\title{
Multiple-option master's degree in optical science and engineering
}

\section{Sudhakar Prasad, John Mclver}

Sudhakar Prasad, John K. Mclver, "Multiple-option master's degree in optical science and engineering," Proc. SPIE 3831, Sixth International Conference on Education and Training in Optics and Photonics, (16 June 2000); doi: $10.1117 / 12.388725$

Event: Education and Training in Optics and Photonics (ETOP'99), 1999, Cancun, Mexico 


\title{
A Multiple-Option Master's Degree in Optical Science and Engineering
}

\author{
Sudhakar Prasad* and John McIver \\ Department of Physics and Astronomy \\ University of New Mexico, Albuquerque, New Mexico 87131
}

\begin{abstract}
The optics faculty at the University of New Mexico (UNM) are proposing to create a Master's degree program in Optical Science and Engineering. A natural complement to the highly successful Ph.D. program in optics over the past 15 years at UNM, the Master's program, unlike the Ph.D. program, will be a multiple-option program that will serve the educational, research, and training needs of an entire spectrum of students, professionals, and institutions. Currently only in the stages of a well developed proposal, it has garnered wide support from industry, academia, and government laboratories in the State of New Mexico, and is on track for an expected implementation in Fall 2000.
\end{abstract}

Keywords: Master's degree, optical science and engineering, multiple-option program

\section{INTRODUCTION}

For the past 15 years, the Departments of Physics and Astronomy and of Electrical and Computer Engineering at the University of New Mexico have administered a highly successful Ph.D. program in Optical Sciences. ${ }^{1}$ During this interval, over $100 \mathrm{Ph}$.D.'s have been awarded to optical physicists and electrical engineers who have become highly valuable members of optical industries and academia here and abroad. During this same period, the optics-related faculty has seen its numerical strength increase to a healthy 20 today.

Over the years, a strong need for a Master's program in Optical Science and Engineering has been felt by the students and faculty alike. A survey of optics students done sometime ago showed an overwhelming support for an M.S. degree. Informal contact with the Los Alamos National Laboratory, Air Force Research Laboratory, and Sandia National Laboratory, all located in New Mexico, has also revealed great interest in such a program. National surveys also indicate a growing need for short-haul specialized training, as provided ideally by the Master's degree rather than the Ph.D. A recent three-year study of the Committee on Optical Science and Engineering (COSE) ${ }^{2}$ instituted by the National Research Council of the National Academies of Science and of Engineering on the state of optics and photonics is unequivocal in its prediction that the need for optics personnel at all levels in industrial settings will easily outstrip their supply from the relatively few institutions that offer specialized optics degrees. In spite of this somewhat alarming situation, there are still only a few Master's programs in optics throughout the country, the most notable being the ones at the Optical Sciences Center, University of Arizona and at the Institute of Optics, University of Rochester.

With the overwhelming evidence to indicate the desirability of such a program, we recently sent out questionnaires to industrial establishments, mostly local but some national as well, to gauge their level of interest and to solicit their input vis-a-vis an optics Master's degree. Judging from their responses the level of enthusiasm seems high. The recent formation of the New Mexico Optics Industry Association (NMOIA), Inc. and its enthusiastic endorsement by organizations like the Albuquerque Economic Development, NM Department of Economic Development, and the Albuquerque Chapter of the Optical Society of America add a new dimension and urgency to the establishment of such a Master's degree program at UNM.

* Correspondence: E-mail - sprasad@unm.edu; Telephone - 505277 5876; Fax - 5052771520 


\section{MOTIVATION FOR THE MS PROGRAM}

The following itemization summarizes the most important reasons for creating an M.S. degree program in Optical Science and Engineering at UNM:

- Fulfilling the need for short-term retraining of scientific staff at industries and national labs;

- Creating a pool of highly trained optics personnel who are readily employable, as revealed by national surveys;

- Creating a more symbiotic partnership between UNM and industries that could result in student support, scholarships, grants, etc. The formation of the NMOIA makes such a partnership extremely viable and indeed vital to the economy of New Mexico;

- Desirability of a Master's degree (relative to a Ph.D. degree) for industry - greater growth potential, broader preparation/education, etc.;

- Serving better the career goals of certain optics Master's degree aspirants who, in the absence of an Optics Master's, are forced to either switch to one of the existing Master's programs in Physics and EECE or even leave the university. Such migration, caused thus by a mismatch of student expectations with the education/training provided by the existing degree programs, diminishes the quality, impact, viability, and visibility of our commitment to Optics education and research as exemplified by the Ph.D. program;

- A potentially strong recruitment tool in the present times of dwindling graduate enrollments nation-wide, that would make us competitive with the Optics MS programs at Arizona, Rochester, and Central Florida in attracting top-notch students;

- Completeness of a major graduate education and research program like Optics, in which both UNM and the State of NM have already invested a great deal, requires that both M.S. and Ph.D. degrees be awarded; and

- No other NM institution of higher learning has either a similar program or extant resources to create one. Yet this program will meet the career goals of certain graduates of these institutions and UNM, who are otherwise likely to leave the state.

Furthermore, the proposed M.S. program would require only minimal extra teaching/administrative load beyond what we have at present.

\section{COURSE WORK, THESIS, AND OTHER REQUIREMENTS}

We cite a major recommendation of the COSE Report: ${ }^{2}$ Universities should encourage multidisciplinarity in optics education, cutting across departmental boundaries, and should provide research opportunities at all levels, from the bachelor's degree to the doctorate and from basic science to applied technology. As if to fulfil this mandate, which we had in a way anticipated by fifteen years when we established our cross-disciplinary Ph.D. program, the proposed M.S. program will be a joint Physics and Astronomy - Electrical and Computer Engineering program in optics and photonics, an area identified as a "bridge" area at a most important, cross-cutting edge of the educational and technological needs of the 21 st century.

The Graduate Coordinators in the two participating departments, Physics and Astronomy and in Electrical Engineering and Computer Engineering, will serve as the administrative contacts for M.S. degree candidates, just as they have for Ph.D. students. Application to the program could be addressed to either Coordinator. The completed files will be evaluated by an Admission Committee culled from among the optics faculty.

This will be a cross-disciplinary program, in which we shall welcome students from a wide range of disciplines, with different backgrounds and levels of experience. We propose to offer a flexible program that 
can effectively accommodate the various levels of preparation, and provide a solid base in optics as well as specialized preparation in the area of research that the student chooses (see the Thesis and Internship Options below). The pace-setting work in ultrafast optics and photonics, high-resolution imaging, quantum optics, optoelectronic devices, fiber amplifiers, optical materials, and novel optical lithography will serve as the focus areas for the Master's program (please see Appendix A for research facilities).

Keeping track of student progress is vital to the success of any academic program. This will be done mainly by the process of regular advisement. Each entering student will undergo an initial advisement to be carried out by a mentor (preferably one so identified by the student) and one permanent member of an advising committee. A Committee on Studies consisting of three faculty, of whom two will be Optics faculty, will be formed soon after the student enrolls. Each graduate student will be required to meet at least once a semester with his or her committee to review the plan of study.

\section{III.1. Course Work}

To account for the diversity of interests in Optics, the required course work for all three plans has been divided into two categories (the syllabi for the courses, each worth 3 credit hours, appear in Appendix B):

\section{Mandatory courses}

The following six courses, with one choice, must be taken by all students:

\section{- Advanced Optics I}

- Laser Physics I

- Optics Lab

- Microelectronics Processing I Lab

- Electromagnetism

- Guided Wave Optics or Optical Fiber Communication

\section{Option-based courses}

Students choose from the following courses to complete the 24 credit hours of (non-seminar) required course work:

- Introduction to Optoelectronics

- Quantum Mechanics I

- Advanced Optics II

- Nonlinear Optics

- Solid State Physics

- Semiconductor Properties

- Topics in Modern Optics

- Special Topics (EECE 595) 
- Laser Physics II

- Semiconductor Lasers and LEDs

- Quantum Optics

- Atomic and Molecular Structure

- Optical Coherence Theory

\section{III.2. The Various Degree Options}

Each student admitted to the M.S. degree program will choose one of the three plans outlined below.

\section{III.2.1. Option 1. Thesis based}

In this option, students must consult with a faculty and pick a topic of research on which to do a Master's thesis. A proposal for this thesis must be made soon after the topic is selected, so that the student's Thesis Committee can approve the topic. When thesis work is complete, a thesis defense must be made by the student in a manner analogous to a Ph.D. dissertation defense.

The minimum requirements for the thesis-based M.S. option include 6 hours of thesis credit and 24 hours of course work for credit drawn from the preceding list of mandatory and option-based courses.

\section{III.2.2. Option 2. Course based}

The minimum requirements for the course-based M.S. option include 33 hours of course work for credit, including 24 hours drawn from the preceding list of mandatory and option-based courses and 3 hours of research seminar/problems course, including 2 hours in Optics.

\section{III.2.3. Option 3. Internship based}

This plan requires an industrial or a national/federal lab internship lasting between six months to a year. Students must interview with participating industry or lab representatives under the guidance of a faculty representative or liaison and arrange an internship with appropriate industry or lab at the industrial or lab site. Short progress reports at regular intervals, as well as a final report, must be submitted by the student to a committee consisting of the faculty liaison, another optics faculty, and the student's industrial or lab contact. Internships would typically follow course work in the first year to a year and a half after the student has entered the program.

Students would need to enroll in a 3-credit-hour course called Internship in Optical Science and Engineering to fulfill the Internship requirement. Creation of this course, to be cross-listed in P\&A and EECE, will proceed along side the approval process for the entire program. The 3-hour Internship requirement is designed to replace the 3-hour research seminar/problems course requirement of Option 2. Otherwise, this Option has exactly the same academic components as Option 2.

An Industrial/Lab Advisory Committee consisting of $R \& D$ representatives from actively participating industry and labs will serve to develop, refine, and oversee this Option in close cooperation with the Optics Graduate Committee. It is also foreseen that certain seminar-type courses will be regularly offered by industrial and lab scientists and engineers to keep the program vibrant and relevant to their needs.

Courses will be taught to accommodate industrial/lab constraints, e.g., via evening classes and remote TV and other "piped" classes. Further details of these constraints and other matters pertaining to the Internship Option, including a "pretraining" course on Safety, will be worked out in close consultation with the Industrial/Lab Advisory Committee. 


\section{III.3. The Master's Oral Exam (for the Non-Thesis Options)}

All students seeking an M.S. degree under Options 2 and 3 must pass an M.S. Oral Exam in Optical Science and Engineering. This exam, to be administered by the student's Committee on Studies, will test the student on material covered in Advanced Optics I, Laser Physics I, Electromagnetic Theory, and GuidedWave Optics/Optical Fiber Communication. The Optics Graduate Committee is the body empowered to make the final decision on pass/fail recommended by the involved Committees on Studies. The student will have at most two attempts at passing the Oral Exam.

\section{III.4. Thesis Defense (for the Thesis Option)}

A satisfactory thesis must be submitted with the individual members of the Thesis Committee after the completion of research, and on their approval the student must defend his thesis work in a pre-advertised presentation to the members of the Thesis Committee and other interested members of the university.

\section{III.5. Advancement to Ph.D. Candidacy}

MS students in the last semester of their MS degree may petition the Optics Graduate Committee to take the Ph.D. Comprehensive Examination in Optical Sciences. Permission will normally be given only if the student meets the qualifications for admission to the Ph.D. program.

\section{III.6. Pre-Requisites to Admission}

The minimum entrance requirements are the same as for the Ph.D. program. The following undergraduate requirements must be met:

- General Physics (3 semesters) consisting of Physics I (Mechanics, sound, fluid mechanics) Physics II (Thermodynamics, electricity and magnetism) Physics III (EM waves, light, special relativity, atoms, molecules, nuclei, and elementary particles)

- Chemistry (2 semesters) covering an introduction to the chemical and physical behavior of matter.

- Electromagnetism (2 semesters) covering electrostatics, solution of electrostatic problems (Laplace and Poisson equations), dielectric media, microscopic theory of dielectrics, electric energy, current, magnetic field (Biot-Savart and Ampere Laws), magnetic materials, electromagnetic induction, magnetic energy, slowly varying (AC) currents, Maxwell's equations, EM waves, waves at boundaries, waveguides, and resonators, optical dispersion, emission from dipoles and antenna, and electrodynamics of moving charges.

- Elementary Quantum Mechanics covering thermal radiation and Planck's Postulate, photons (photoelectric and Compton effects, pair production and annihilation), Heisenberg uncertainty principle, postulates of quantum mechanics, one-dimensional potentials, angular momentum, H-atom.

- Mathematics requirement - Calculus (3 semesters); Vector analysis (1 semester); Ordinary Differential Equations (1 semester); Partial Differential Equations (1 semester).

- Knowledge of elementary optics is not required for entrance, but is a pre-requisite for the core course Advanced Optics I. 


\section{CONCLUDING REMARKS}

The Master's degree in Optical Science and Engineering aims at creating a unique and highly desired cadre of optics personnel who, because of their broad, advanced, interdisciplinary, and industrially relevant education and training, will be scientific and industrial leaders in the optics and photonics engaged 21st century. Graduates of this program will display unique adaptability to changing modern industrial and scientific needs, will have entrepreneurial skills, and will be members of a highly effective work force in optics and photonics.

The proposed program is expected to be offered beginning Fall 2000.

\section{ACKNOWLEDGMENTS}

In developing the MS proposal described in this article, the authors have received invaluable help from a variety of people and organizations. We have benefited greatly, in particular, from the comments of S. Brueck, J.-C. Diels, M. Fischer, P. Fleury, A. Guenther, R. Jain, L. Lester, M. Osinski, W. Rudolph, and M. Sheik-Bahae, all of UNM; L. Pedrotti of CORD; and B. Hunter, B. Leasure, and K. Lear, all of NMOIA.

\section{REFERENCES}

1. The Ph.D. program in Optical Sciences at UNM has been described in a variety of easily available sources. Among them are:

a. SPIE Optics Education 2000 Catalog of world-wide educational programs, front cover, inside front cover, and p. 46; also available on the web at http://www.spie.org/web/oped;

b. M. Sheik-Bahae, "Research and education in optics and photonics at the University of New Mexico," LEOS Newsletter, December 1998;

c. S. Prasad and A. Guenther, "Optics is for Seeing Clearly: The University of New Mexico Story," SPIE newsletter, OE Reports, to be published August 1999; and

d. Our web site, http://www.optics.unm.edu.

2. Harnessing Light: Optical Science and Engineering for the 21st Century, National Research Council's COSE Report, (National Academy Press, Summer 1998). 


\section{APPENDIX A: FACILITIES AND ASSOCIATED INSTITUTIONS}

Optics research at the University of New Mexico has acquired an international reputation over the last decade. Pioneering work has originated from this program in areas ranging from the quantum theory of lasers, squeezed states, and correlated emission lasers, to practical applications such as surface emitting semiconductor lasers, new material developments (PLZT thin films) for nonlinear optics, ultrashort pulse physics, and new concepts of laser gyros, to cite only a few recent examples.

The large externally sponsored research activity within the Optics Program has nurtured the creation and development of well equipped Optics Instruction Laboratories, in which graduate students get acquainted with most of the tools to be used in their thesis work. Examples of Optics Laboratory experiments are linear optics, Gaussian optics, Fourier optics, statistical optics, Doppler velocimetry, self focusing of Argon laser beams, measurement of nonlinear index by z-scan techniques, correlation techniques, femtosecond techniques, and measurement of dispersion by white light interferometry.

We do not operate in an intellectual vacuum, but enjoy the proximity of Los Alamos National Laboratory, Sandia National Laboratories, and the Air Force Research Laboratory. Albuquerque is also home to a growing number of semiconductor manufacturing facilities including Intel, Philips Semiconductors, and Motorola. Close collaboration with these institutions takes the form of contracts, joint appointments, and student supervision for work performed at these sites.

Through fast fiber-optic links, optics graduate students have access to high speed computers and clusters and to massively parallel computation. Through a cooperative agreement with the US Air Force Research Laboratory (AFRL), UNM manages and coordinates computational activities at the premier Maui HighPerformance Computing Center (MHPCC). UNM has established locally the Albuquerque High Performance Computing Center for parallel code development and scientific computation that can be ported to the MHPCC supercluster.

Students will also find a well equipped machine shop with a very friendly staff, to help with equipment design and fabrication.

Two main research Centers interact with the Optics program, namely the Center for High Technology Materials (CHTM) and the Center for Advanced Studies (CAS). The former (CHTM) is primarily an experimental research center, providing a link between the University and Industry. The latter (CAS) is primarily a theoretical "think tank", preparing the path for future applications and technology.

CHTM, closely connected with the EECE department, is oriented towards optoelectronic and semiconductor research. Facilities include class-100 clean rooms, Metal-Organic Chemical-Vapor Deposition (MOCVD) and Liquid-Phase Epitaxy (LPE), submicron device fabrication, and extensive device characterization, modeling and analysis. The analysis capabilities include Scanning Electron Microscope (SEM), Scanning Tunneling Electron Microscope (STEM), Transmission Electron Microscope (TEM), and various field emission spectroscopies. More specific to the optics program are ultrafast (ns, ps and fs) linear and nonlinear spectroscopies, optical thin film research, and development of new nonlinear materials.

CAS, housed in the Physics and Astronomy department, is an institute of theoretical optics and condensedmatter physics, but is rapidly evolving toward a truly cross-disciplinary status that involves researchers from five departments, Physics and Astronomy, Mathematics, Chemistry, Geology, and Biology. In addition to a productive research activity (in quantum optics, quantum measurement theory, interferometric imaging, information physics, exciton and charge transport in materials), CAS has a prestigious seminar series with speakers of international reputation (DeMartini, Walther, Kimble, Gell-Mann, Yamamoto, etc.).

Optics research extends beyond the Electrical and Computer Engineering and Physics and Astronomy departments. There are close collaborations between faculty and students of the Optics Program, the Department of Chemistry, the Department of Biology and the UNM Cancer Center. 


\section{APPENDIX B: COURSE SYLLABI}

- Advanced Optics I: Electromagnetic theory of geometrical optics, Gaussian ray tracing and matrix methods, finite ray tracing, aberrations, interference, and diffraction.

This course is identical to the course of Optics I presently offered for the Ph.D. program in Optics.

- Electromagnetism: Maxwell's equation, wave propagation in vacuum and in homogeneous media, elements of electrostatics, Green's function, light-matter interaction, dipole radiation, waveguides, scattering.

- Laser Physics I: Quantum theory of radiation, introduction to two-level system, spontaneous and stimulated emission, rate equation, gas, semiconductor, and solid-state lasers.

This course is identical to the course of "Laser physics I" presently offered for the Ph.D. program in Optics.

- Optics Lab: Series of experiments illustrating Optics I and Laser Physics.

This course is identical to the course of Optics Lab presently offered for the Ph.D. program in Optics.

- Microelectronic Processing I Lab: Process technology for microelectronics, including materials science of semiconductors, device/circuit fabrication, parasitics, and packaging. Lab project features small-group design, fabrication, and testing of a simple MOS circuit.

- Guided-Wave Optics: Optical propagation in free space, colored dielectrics, metals, semiconductors, crystals, graded index media. Radiation and guided modes in complex structures. Input and output coupling, cross-coupling mode conversion. Directional couplers, modulators, sources, and detectors.

- Optical Fiber Communication: Optical waveguiding in uniform optical fibers having step- and gradedindex profiles. Effects of bends, core nonuniformities and other perturbations. Communication system architectures: direct, heterodyne, and homodyne detection. System measurements and optical fiber sensing.

- Introduction to Opto-electronics: Basic electro-optics and opto-electronics, with engineering applications. Interaction of light with matter. Introduction to optics of dielectrics, metals and crystals. Introductory descriptions of electro-optic, acousto-optic, and magneto-optic effects and related devices. Light sources, displays and detectors. Elementary theory and applications of lasers, optical waveguides and fibers.

- Quantum Mechanics I: Review of one-dimensional potentials; Dirac formalism; postulates; symmetries and conservation laws; harmonic oscillator; angular momentum and spin; central potentials; approximation methods.

- Advanced Optics II: Coherence theory, fully coherent objects, imaging by partially coherent light, partially polarized light.

- Nonlinear Optics: General concepts, microscopic approach, transient response and pulse propagation, nonlinear processes.

- Solid State Physics: Free electron gas, energy bands, crystals, semiconductors, metals, elementary excitations, superconductivity.

- Semiconductor Properties: Quantum mechanics and statistical mechanics concepts. Equilibrium and non-equilibrium carrier statistics. Carrier recombination processes. Drift and diffusion. Elemental and compound semiconductors.

- Topics in Modern Optics: Possible topics include dye lasers, solid-state lasers, novel lasers, interaction between intense lasers and matter, advanced nonlinear optics spectroscopy. 
- Special Topics in EECE:

- Laser Physics II: Semiclassical laser theory, mode problems, pulse propagation, self-induced transparency, phase conjugate optics, photon statistics.

- Semiconductor Lasers and LEDs:

- Quantum Optics: Photon statistics, superradiance, advanced laser theory, quantum noise, quantum non demolition, and the application of quantum optical techniques to the foundations of physics.

- Atomic and Molecular Structure: One-, two-, and many-electron atoms; interactions with electromagnetic radiation; fine and superfine structure; external fields; molecular structure and spectra; collisions; applications of atomic and molecular physics.

- Optical Coherence Theory: Time dependence of coherent and incoherent light beams, intensity fluctuations of chaotic light, fringe intensity, first-order correlation function, higher-order correlation functions, photoelectron statistics. 